\title{
Red Shift of Faraday Rotation in Thin Films of Completely Bismuth-Substituted Iron Garnet $\mathrm{Bi}_{3} \mathrm{Fe}_{5} \mathrm{O}_{12}$
}

\author{
Ming-Yau Chern, Fang-Yuh Lo, Da-Ren LiU, Kuang YANG and Juin-Sen LiAw \\ Department of Physics, National Taiwan University, Taipei, Taiwan, R.O.C.
}

(Received June 17, 1999; accepted for publication August 31, 1999)

The magnetooptical Faraday rotations of epitaxial films of $\mathrm{Bi}_{x} \mathrm{Y}_{3-x} \mathrm{Fe}_{5} \mathrm{O}_{12}$ (Bi:YIG) grown on [111]-oriented gadolinium gallium garnet (GGG) substrates by pulsed laser deposition (PLD) were studied with bismuth content $x=0.5,1.0,1.5,2.0$, 2.5, and 3.0. The Faraday rotation angles, $\theta_{\mathrm{F}}$, of the films were measured by the method of rotating analyzer ellipsometry (RAE) with the photon energy varied from 1.5 to $3.5 \mathrm{eV}$. It was shown that in addition to the increase of the Faraday rotation with increasing $x$, the peaks of $\theta_{\mathrm{F}}$ shifted toward the red region as $x$ changed from 1.0 to 1.5. The peak positions of $\theta_{\mathrm{F}}$ for the completely Bi-substituted iron garnet, $\mathrm{Bi}_{3} \mathrm{Fe}_{5} \mathrm{O}_{12}$ (BIG), were found at 2.4 and $2.8 \mathrm{eV}$ with peak values as large as $-23 \mathrm{deg} / \mu \mathrm{m}$ and $44 \mathrm{deg} / \mu \mathrm{m}$, respectively

KEYWORDS: magnetooptical Faraday rotation spectra, $\mathrm{Bi}_{3} \mathrm{Fe}_{5} \mathrm{O}_{12}, \mathrm{BIG}, \mathrm{Bi}_{x} \mathrm{Y}_{3-x} \mathrm{Fe}_{5} \mathrm{O}_{12}, \mathrm{Bi}: \mathrm{YIG}$, pulsed laser deposition, PLD

\section{Introduction}

It is well known that when the yttrium iron garnet $\mathrm{Y}_{3} \mathrm{Fe}_{5} \mathrm{O}_{12}$ (YIG) is substituted with bismuth, the magnetooptical (MO) effects of bismuth-doped compounds, $\mathrm{Bi}_{x} \mathrm{Y}_{3-x} \mathrm{Fe}_{5} \mathrm{O}_{12}$ (Bi:YIG), are strongly enhanced. ${ }^{1)}$ The Faraday optical spectra of Bi:YIG films have been subjected to extensive studies in order to understand the microscopic origin of the large MO effects. ${ }^{2-8)}$ To obtain the maximal effects in this system and to fully understand the system, it is necessary to synthesize the completely Bi-substituted iron garnet, $\mathrm{Bi}_{3} \mathrm{Fe}_{5} \mathrm{O}_{12}$ (BIG); however, due to thermodynamics, $\mathrm{Bi}$ incorporation in the LPE films is limited to about two atoms per formula unit. ${ }^{9)}$ Since samples with high Bi content were relatively difficult to prepare, ${ }^{10-12)}$ very few works on the Faraday rotation spectrum and the Curie temperature of BIG films were reported. ${ }^{10,11)}$ In this paper, we report the measurements of Faraday rotation spectra and Curie temperatures of a series of epitaxial Bi:YIG films grown on [111]-oriented $\mathrm{Gd}_{3} \mathrm{Ga}_{5} \mathrm{O}_{12}$ (GGG) substrates by pulsed laser deposition (PLD) with $\mathrm{Bi}$ incorporation of up to three atoms per formula unit.

\section{Experimental}

Details regarding the synthesis and characterization of Bi:YIG films have been described previously, ${ }^{13)}$ and only the most relevant are given here. The films were deposited using an ArF excimer laser with an energy of $200 \mathrm{~mJ}$ per pulse at a repetition rate of $4 \mathrm{~Hz}$ and a fluence of $2 \mathrm{~J} / \mathrm{cm}^{2}$ at the target. The Bi:YIG targets were sintered polycrystalline pellets of the composition $x \mathrm{Bi}_{2} \mathrm{O}_{3}+(3-x) \mathrm{Y}_{2} \mathrm{O}_{3}+5 \mathrm{Fe}_{2} \mathrm{O}_{3}$, where $x=0.5,1.0,1.5,2.0,2.5$, and 3.0. The [111]GGG substrates were placed on a heated holder positioned $5 \mathrm{~cm}$ from the target. An oxygen flow of $20 \mathrm{sccm}$ was introduced to the growth chamber, and oxygen pressure in the chamber was maintained at 200 mTorr during deposition. Since the amount of material deposited for each laser pulse was approximately constant, the thickness of the films was controlled by counting the number of laser pulses. The thickness of the films was $\sim 0.3 \mu \mathrm{m}$ for 25,000 laser pulses, as calibrated by highresolution X-ray rocking curves. In general, the temperature for the non-equilibrium epitaxial growth of Bi:YIG films decreases as the content of bismuth increases, which is consistent with the report on the reduction of the preparation tem- perature of polycrystalline garnets by bismuth substitution. ${ }^{14)}$ For $x=0.5$, the most suitable growth temperature in terms of crystal structure and Curie temperature is around $650^{\circ} \mathrm{C}$; however, for $x=3,500^{\circ} \mathrm{C}$ is the best. An energy dispersive $\mathrm{X}$-ray (EDX) analysis confirmed that the Bi composition $x$ of the films is the same as that of the targets within experimental error.

The Faraday rotations of the samples were measured using the technique of rotating analyzer ellipsometry (RAE). ${ }^{15)}$ The output from a $75 \mathrm{~W}$ xenon lamp was guided by an optical fiber and then focused onto the samples, to which a magnetic field was applied perpendicularly. The light was polarized by a calcite Glan-Taylor prism before irradiating the samples. The polarization of the light exiting from the samples was analyzed by another calcite prism polarizer, which was rotated by a computer controlled stepping motor. The saturation Faraday rotations under a magnetic field of $1.5 \mathrm{~T}$ were analyzed at $18^{\circ}$ intervals while the analyzing prism was rotated. The Faraday rotation angles were then calculated using a Fourier transform procedure. ${ }^{15)}$ Since the samples absorb UV light above $3.5 \mathrm{eV}$, the rotation spectra were taken from 1.5 only up to $3.5 \mathrm{eV}$ by scanning a monochromator. Care was taken to eliminate the combined polarization effect of the monochromator and the photomultiplier detector. Since the GGG substrate introduces a small Faraday rotation, it was necessary to subtract the contribution from the total Faraday rotation to obtain the true rotation of the films.

The Curie temperatures of the films were measured using a new magnetooptical technique. A small ac magnetic filed, $H_{\text {rms }} \sim 50 \mathrm{mG}$, was applied to the samples, and then the polarization of the linearly polarized light passing through the samples was analyzed while a sweeping dc field from $-10 \mathrm{G}$ to $+10 \mathrm{G}$ was superimposed. Below $T_{\mathrm{c}}$, for each scan of the dc field, there was a sharp peak of the ac MO signal around zero dc field, which corresponded to the maximum of the susceptibility $\chi(=\mathrm{d} M / \mathrm{d} H)$. The Curie temperature was determined as the temperature where the peak of the ac signal vanished. This technique, the details of which will be published elsewhere, ${ }^{16)}$ is sufficiently sensitive to unambiguously determine $T_{\mathrm{c}}$ to within $0.5^{\circ} \mathrm{C}$.

\section{Results and Discussion}

The high resolution $\mathrm{X}$-ray rocking curve of sample $\mathrm{Bi}_{0.5} \mathrm{Y}_{2.5} \mathrm{Fe}_{5} \mathrm{O}_{12}$ is shown in Fig. 1. A well-matched sim- 


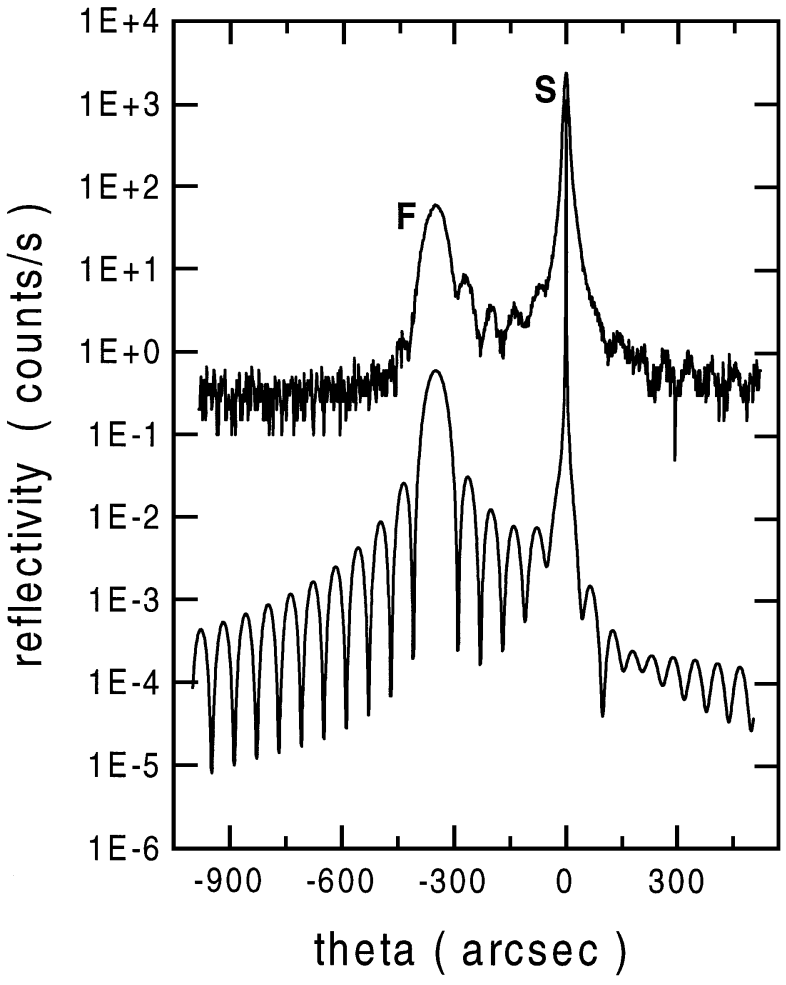

Fig. 1. High-resolution X-ray rocking curve of (444) reflections of $\mathrm{Bi}_{0.5} \mathrm{Y}_{2.5} \mathrm{Fe}_{5} \mathrm{O}_{12}$ film (F) on [111]GGG substrate $(\mathrm{S})$. Simulation of the rocking curve is also shown but shifted down by two decades for comparison.

ulation $^{17)}$ of the rocking curve is achieved using $12.443 \AA$, $0.298 \mu \mathrm{m}$, and 0.29 as the lattice constant, film thickness, and the Poisson ratio, respectively. The oscillatory fringes near the main diffraction peaks ( $\mathrm{F}$ and $\mathrm{S}$ ) and the almost identical line widths (FWHM $\sim 56$ arcsec for the film peak F compared to $\sim 52$ arcsec for the theoretical width from simulation) indicate that the epitaxial films are of high crystalline quality. For $x>1.0$, the diffraction peaks become broad, indicating the formation of defects. ${ }^{13)}$

The Curie temperatures of the Bi:YIG samples and the undoped YIG $(x=0)$ films grown also by PLD are plotted in Fig. 2 to show their relationship with Bi content $x$. Although, as expected, $T_{\mathrm{c}}$ increases as $x$ increases, there is an obvious discontinuity of the trend between $x=1.0$ and 1.5. A nonlinear behavior of the trend is obvious from the plot; therefore, for the first-order approximation, quadratic fits were used to connect data points at $x=0,0.5$, and 1.0 and $x=0,1.5$, 2.0, 2.5, and 3.0. Initially, for $x=0,0.5$ and 1.0, the slope $\Delta T_{\mathrm{c}} / \Delta x$ of the fit is not a constant but decreases as $x$ increases, and then there is a jump of $T_{\mathrm{c}}$ at $x=1.5$. From $x=1.5$ to $3.0, T_{\mathrm{c}}$ is back to the dashed line connecting the points at $x=0,1.5,2.0,2.5$, and 3.0. The initial slope $\Delta T_{\mathrm{c}} / \Delta x$ of the fitting curve turns out to be $\sim 38^{\circ} \mathrm{C} / x$, which is consistent with the value for LPE films. ${ }^{18)}$ Finally, $T_{\mathrm{c}}$ deviates from the linear relationship with $x$ and levels off as Bi content becomes saturated, i.e. $x=3.0$.

The behavior of $T_{\mathrm{c}}$ can be explained by the discontinuity of the lattice constants shown in the inset in Fig. 2 discussed previously. ${ }^{13)}$ The incorporation of $\mathrm{Bi}$ expands the YIG lattice; thus, for $x=0.5$ and 1.0, the films are under compressive stress and the lattice constants perpendicular to the film, $a_{\perp}$,

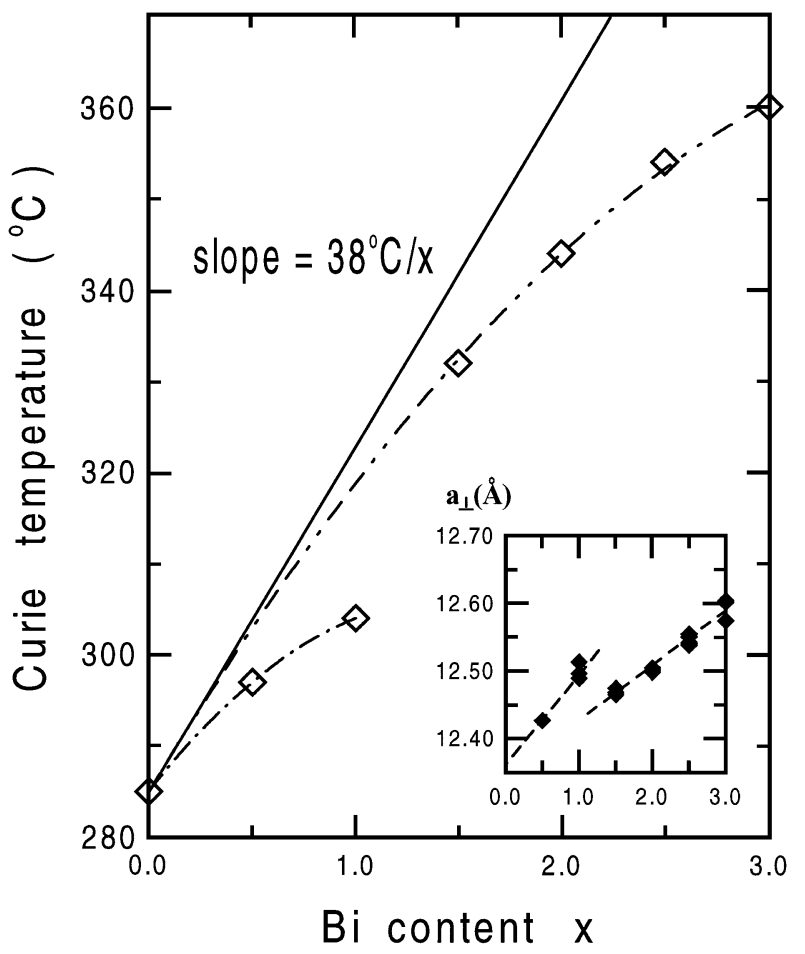

Fig. 2. Plot of the Curie temperatures of the Bi:YIG films as a function of Bi content $x$. Dashed lines are quadratic fits to the data points and the solid line is the slope $\Delta T_{\mathrm{c}} / \Delta x$ at $x=0$ of the fitting curve connecting the data for $x=0,1.5,2.0,2.5$ and 3.0 (skipping $x=0.5$ and 1.0). The perpendicular lattice constants $a_{\perp}$ of the films, taken from our previous report, ${ }^{13)}$ are also shown in the inset for comparison.

are larger than their bulk values. However, for $x>1.0$, the lattice mismatch becomes so large that the films relax. $T_{\mathrm{c}}$ is lowered by the compressive stress at $x=0.5$ and 1.0 ; in contrast, $T_{\mathrm{c}}$ shows a break at $x=1.5$ when the film relaxes. The lowering of $T_{\mathrm{c}}$ for higher $x$ might be due to the higher defect density in Bi:YIG lattices with higher Bi concentration. ${ }^{13)}$

The Faraday rotation spectra from 1.5 to $3.5 \mathrm{eV}$ of the Bi:YIG samples are shown in Fig. 3, where the right-handed rule was used to define the sense of rotation. All the spectra show two distinct regions of negative rotation at longer wavelength and positive rotation at shorter wavelength. The magnitude of the spectra increases when Bi content $x$ increases, and, as expected, it reaches the highest values, $-23 \mathrm{deg} / \mu \mathrm{m}$ and $+44 \mathrm{deg} / \mu \mathrm{m}$ for the two regions when $x=3.0$. The most interesting finding is that both of the maximal rotations in the two regions shift toward longer wavelengths when $x$ is larger than 1.0. For $x=0.5$ and 1.0, the peaks of the spectra occur at 2.6 and $3.2 \mathrm{eV}$, as indicated by letters $\mathbf{b}$ and $\mathbf{d}$ in Fig. 3, respectively. However, for $x=1.5,2.0,2.5$ and 3.0, the peaks of the spectra shift to 2.4 and $2.8 \mathrm{eV}$, as indicated by letters a and $\mathbf{c}$, respectively.

There have been intensive theoretical studies to elucidate the origin of the giant MO effect in Bi:YIG systems. ${ }^{2-9)}$ Most of the authors agreed that the effect originates from the splitting of the excited states of the $\mathrm{Fe}^{3+}$ ion by the spin-orbital interaction that is enhanced by the mixing of $\mathrm{Bi}^{3+}$ orbitals. However, the available MO active transitions with transition energies in the visible spectrum are still not well resolved. Our data show that at least four possible transitions are required to account for the Faraday rotation spectra, namely, 


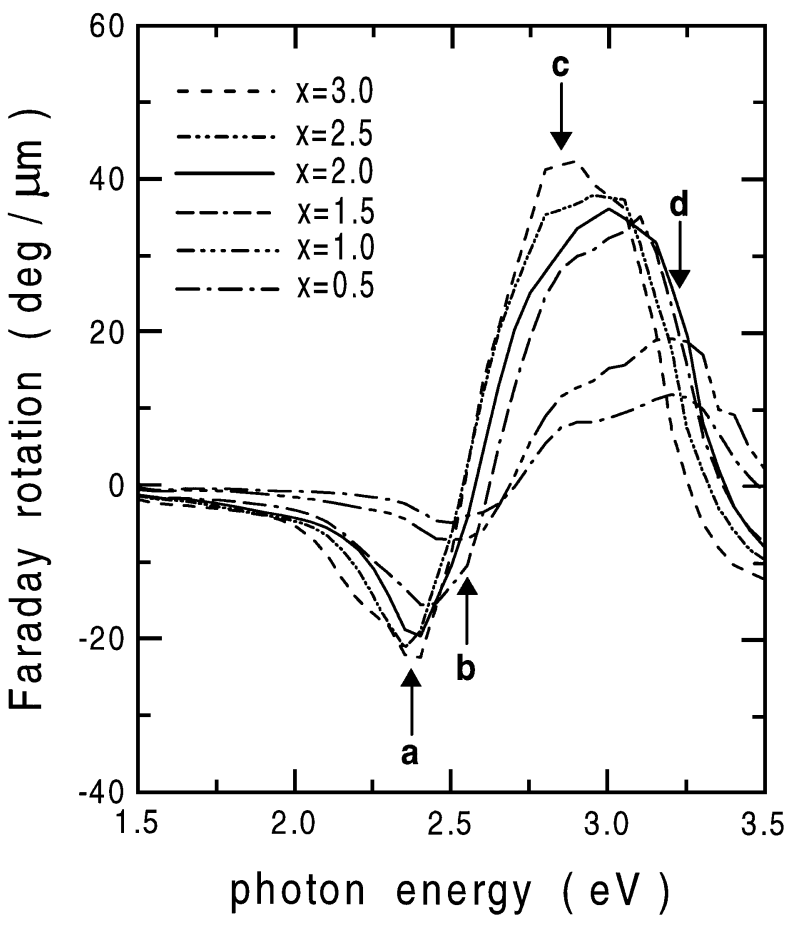

Fig. 3. Faraday rotation spectra of Bi:YIG films showing negative and positive rotations from 1.5 to $3.5 \mathrm{eV}$. The spectra clearly show a red shift, indicated by the letters: $\mathbf{b} \rightarrow \mathbf{a}$, and $\mathbf{d} \rightarrow \mathbf{c}$, when $x$ is larger than 1.0. The overall peaks of the spectra are found at 2.4 and $2.8 \mathrm{eV}$ for $x=3.0$ with very large values of $-23 \mathrm{deg} / \mu \mathrm{m}$ and $+44 \mathrm{deg} / \mu \mathrm{m}$, respectively.

the positions indicated by $\mathbf{a}, \mathbf{b}, \mathbf{c}$, and $\mathbf{d}$ in Fig. 3. Presumably, negative (a and b) and positive (c and $\mathbf{d}$ ) rotations come from the transitions in octahedral and tetrahedral $\mathrm{Fe}^{3+}$ ions with opposing spin magnetic moments in ferrimagnetic $\mathrm{Bi}$ :YIG systems. The shift from $\mathbf{b}$ to $\mathbf{a}$ is a phenomenon reported and discussed before, where $\mathbf{a}$ and $\mathbf{b}$ were assigned as paramagnetic and diamagnetic transitions around $2.5 \mathrm{eV}$, respectively. ${ }^{5)}$ As the result of $\mathrm{Bi}$ incorporation, transition a outgrows transition $\mathbf{b}$, whence the peak shifts from $\mathbf{b}$ to $\mathbf{a} .^{5 \text { ) }}$ From the discontinuities of the Curie temperatures as well as the lattice constants caused by relaxation of the strain, it may be also reasonable to argue that the "discontinuous" red shift of the transitions occurring between $x=1.0$, and 1.5 is probably caused by the relaxation of the lattices as well. Thus, we may claim that transitions $\mathbf{b}$ and $\mathbf{d}$ are enhanced by elongation of the lattice along the [111] direction, whereas transitions $\mathbf{a}$ and $\mathbf{c}$ are favored by the unstrained cubic lattice; however more detailed theoretical consideration and experimental data are required to justify the proposed mechanism along this line.

\section{Conclusions}

In conclusion, we have measured the Faraday rotation spectra and the Curie temperatures of a series of epitaxial $\mathrm{Bi}$-substituted yttrium iron garnet, $\mathrm{Bi}_{x} \mathrm{Y}_{3-x} \mathrm{Fe}_{5} \mathrm{O}_{12}$, films grown on [111]GGG by the non-equilibrium process of pulsed laser deposition. Both the Curie temperatures and the Faraday rotation spectra show abrupt discontinuities between $x=1.0$ and 1.5 due to the change of the lattices from strained to relaxed states. The shift of the maximum of the Faraday spectra from $\mathrm{UV}(3.2 \mathrm{eV})$ to the blue $(2.8 \mathrm{eV})$ region may have advantages in applications related to recent developments of blue semiconductor laser technology. It would be very interesting to further test the effects of strain on the MO properties of the films by using other garnet substrates with different lattice constants.

\section{Acknowledgments}

This work was supported by the National Science Council of the Republic of China.

1) D. E. Lacklison, G. B. Scott, H. I. Ralph and J. L. Page: IEEE Trans. Magn. 9 (1973) 457.

2) S. Wittekoek, T. J. A. Popma, J. M. Robertson and P. F. Bongers: Phys. Rev. B 12 (1975) 2777.

3) Z. Simsa, H. Le Gall, J. Simsova, J. Kolacek and A. Le Paillier-Malecot: IEEE Trans. Magn. 20 (1984) 1001.

4) V. Doormann, J.-P. Krumme and H. Lenz: J. Appl. Phys. 68 (1990) 3544.

5) K. Matsumoto, S. Sasaki, K. Haraga, K. Yamaguchi and T. Fujii: IEEE Trans. Magn. 28 (1992) 2985.

6) G. F. Dionne and G. A. Allen: J. Appl. Phys. 73 (1993) 6127.

7) G. F. Dionne and G. A. Allen: J. Appl. Phys. 75 (1994) 6372.

8) J. Wei, H. Hu and H. He: Phys. Status Solidi A 168 (1998) 501

9) P. Hansen, W. Tolksdorf, K. Witter and J. M. Robertson: IEEE Trans. Magn. 20 (1984) 1099.

10) A. Thavendrarajah, M. Pardavi-Horvath, P. E. Wigen and M. Gomi: IEEE Trans. Magn. 25 (1989) 4015.

11) T. Okuda, T. Katayama, K. Satoh and H. Yamamoto: J. Appl. Phys. 69 (1991) 4580.

12) S. Mino, M. Matsuoka, A. Tate, A. Shibukawa and K. Ono: Jpn. J. Appl. Phys. 31 (1992) 1786.

13) M. Y. Chern and J. S. Liaw: Jpn. J. Appl. Phys. 36 (1997) 1049.

14) S. Geller, H. J. Williams, G. P. Espinosa, R. C. Sherwood and M. A. Gilleo: Appl. Phys. Lett. 3 (1963) 21.

15) W. Budde: Appl. Opt. 1 (1962) 201.

16) M. Y. Chern, D. R. Liu, K. Yang and F. Y. Lo: in preparation.

17) Program RADS, Bede Scientific Instruments Ltd., 1994.

18) P. Hansen, K. Witter and W. Tolksdorf: Phys. Rev. B 27 (1983) 6608. 